\section{Effects of antidepressant treatment following} myocardial infarction ${ }^{\dagger}$

\author{
JOOST P. VAN MELLE, PETER DE JONGE, ADRIAAN HONIG, \\ AART H. SCHENE, ASTRID M. G. KUYPER, HARRY J. G. M. CRIJNS, \\ ANNIQUE SCHINS, DORIEN TULNER, MAARTEN P. VAN DEN BERG \\ and JOHAN ORMEL on behalf of the MIND-IT investigators
}

\section{Background Depression following myocardial infarction is associated with poor cardiac prognosis. It is unclear whether antidepressant treatment improves long-term depression status and cardiac prognosis.}

\begin{abstract}
Aims To evaluate the effects of antidepressant treatment compared with usual care in an effectiveness study.
\end{abstract}

\begin{abstract}
Method In a multicentre randomised controlled trial, 2177 myocardial infarction patients were evaluated for ICD-10 depression and randomised to intervention ( $n=209$ ) or care as usual $(n=122)$. Both arms were evaluated at 18 months post-myocardial infarction for long-term depression status and new cardiac events.
\end{abstract}

\begin{abstract}
Results No differences were observed between intervention and control groups in mean scores on the Beck Depression Inventory (II.0, s.d. $=7.5$ v. I0.2, s.d. $=5.1$, $P=0.45)$ or presence of ICD -10 depression (30.5 v. 32.1\%, $P=0.68)$. The cardiac event rate was $14 \%$ among the intervention group and $13 \%$ among controls (OR=1.07, 95\% Cl 0.57-2.00).
\end{abstract}

Conclusions Antidepressant treatment did not alter long-term depression post-myocardial infarction status or improve cardiac prognosis.

Declaration of interest None. Funding detailed in Acknowledgements.

†See invited commentary, pp. 467-468, this issue.
Depression is one of the most potent psychosocial risk factors for a poor cardiovascular prognosis after myocardial infarction (Hemingway \& Marmot, 1999). A recent meta-analysis (Van Melle et al, 2004) showed that depression postmyocardial infarction was associated with a 2- to 2.5-fold increased risk for all-cause mortality, cardiovascular mortality and cardiovascular events. In addition, depression post-myocardial infarction is a major cause of incomplete recovery (Ladwig et $a l, 1994)$, poor quality of life (Beck et al, 2001), delayed return to work (Soderman et al, 2003), non-adherence (Carney et al, 1995), and non-attendance at cardiac rehabilitation (Lane et al, 2001). It is estimated that approximately 1 out of 5 patients has depression post-myocardial infarction (Schleifer et al, 1989).

Two intervention studies have assessed the effects of treatment of depression postmyocardial infarction. In the ENhancing Recovery in Coronary Heart Disease study (ENRICHD), the effects of cognitivebehavioural therapy (CBT) on depression and cardiac prognosis was evaluated (Berkman et al, 2003). In this large trial, no significant difference in cardiac outcomes was found between the intervention and the care as usual arms. Although substantial improvement in depression status was observed 6 months after initiation of CBT, the difference between the arms diminished over time and was no longer present after 30 months.

The SADHART study (Glassman et al, 2002) found sertraline to be a safe treatment for depression post-myocardial infarction, but there was little difference in depression status between groups receiving sertraline and placebo after 24 weeks of treatment. However, the effect of sertraline was greater in the patients with severe and recurrent depression. The study was not designed to assess the effects of treatment on cardiovascular prognosis, but severe cardiovascular events during the 6-month treatment tended to be less frequent in the sertraline group. The effects of sertraline on long-term depression status were not evaluated.

Thus, little is known about the effects of treatment for depression post-myocardial infarction on either long-term depression status or cardiac prognosis. We have conducted the Myocardial INfarction and Depression - Intervention Trial (MIND-IT) in order to determine, using a randomised controlled design, whether antidepressant treatment for depression post-myocardial infarction improves long-term depression status and cardiovascular prognosis (Van den Brink et al, 2002). The MIND-IT is an effectiveness study rather than an efficacy study, and compares the effects of an active treatment strategy with usual care.

\section{METHOD}

\section{Study participants}

The inclusion and exclusion criteria for this trial have been described previously (Van den Brink et al, 2002). In brief, consecutive patients (September 1999 to November 2002) hospitalised for acute myocardial infarction were recruited from ten hospitals (including three tertiary centres) located in different parts of The Netherlands. Patients were enrolled if they met the World Health Organization Multinational Monitoring of Trends and Determinants in Cardiovascular Disease (WHO MONICA) criteria (Gillum et al, 1984) for definite myocardial infarction: increased cardiac enzymes and either electrocardiographic changes or chest pain. Patients were excluded if the index myocardial infarction occurred during a hospitalisation for another medical condition (except for unstable angina pectoris), if they were unable to participate in study procedures (e.g. unable to communicate or not available for follow-up), had any other disease likely to influence short-term survival, were already receiving psychiatric treatment for depression, or were participating in another clinical trial.

The institutional review board at each clinical centre approved the study protocol and all patients provided written informed consent before enrolment. In the study information pack it was emphasised that, although all participating patients were to be screened for depression, antidepressant treatment would be offered only to a random sample of patients and all were free to seek help for mood problems. 


\section{Design of the study}

Patients admitted with an acute myocardial infarction were screened for depressive symptoms during hospitalisation and at 3 , 6,9 and 12 months post-myocardial infarction, using the Beck Depression Inventory (BDI; Beck, 1979). Those with depressive symptoms (i.e. BDI score $\geqslant 10$ ) underwent a psychiatric evaluation using the WHO Composite International Diagnostic Interview (CIDI auto version 2.1; World Health Organization, 1990). The first CIDI interviews were performed at least 3 months post-myocardial infarction to allow natural recovery of depressive symptoms following a major life event. Both screening tools are widely used and their feasibility and reliability have been described elsewhere (Robins et al, 1988; Strik et al, 2001). Patients with a research diagnosis of 'current depressive episode' (World Health Organization, 1993) according to ICD-10 (further: 'depression') were randomised $(1: 1)$ to antidepressant treatment or care as usual. The assignment was carried out at the Trial Coordination Centre in Groningen with the use of computer-generated permuted blocks of four, stratified according to clinical site and time of onset of depression (within 6 months $v .6$ months or more post-myocardial infarction). Because the number of patients actually treated with antidepressants was lower than expected, the randomisation ratio was changed to 2:1 on 14 March 2001. Patients with a significant risk of suicide were excluded from randomisation and referred for treatment outside the study. To compare both strategies, we used the Zelen design (Zelen, 1979): patients allocated to the 'care as usual' arm were not informed about their research diagnosis of depression to avoid influencing usual care. Data management was independently performed at the Trial Coordination Centre, Groningen, The Netherlands.

\section{Baseline variables}

Data were collected on demographics, medical history, clinical variables and medication use during hospitalisation for the index myocardial infarction. The cumulative burden of medical comorbidity was assessed with a modified version of the Charlson Comorbidity Index (Watkins et al, 2003). Higher scores on this scale indicate more comorbidity. To account for a possible relationship between depression post-myocardial infarction and cardiac disease severity, the following parameters of risk stratification were assessed: Killip class at admission, maximum values of serum aspartate transaminase during hospitalisation, left ventricular ejection fraction (as measured by either echocardiography or radionuclide ventriculography) and wall motion score index (WMSI) according to the recommendations of the American Society of Echocardiography (Schiller et al, 1989). Independent analysis was performed at the core echocardiography laboratory by technicians who were unaware of the patients' randomisation status.

\section{Antidepressant intervention}

The MIND-IT study was designed as an effectiveness study comparing active antidepressant treatment with usual care. In the intervention arm, the research diagnosis provided by the CIDI interview was confirmed by a psychiatrist prior to the patient starting antidepressant treatment. Several treatment modalities were possible. Flexibility in treatment was permitted because the main research question was whether implementing any active depression treatment strategy would be associated with better outcomes than usual care in which antidepressant treatment is almost negligible (Frasure-Smith et al, 1993). However, allocation to these modalities was strictly defined in the protocol. First-choice treatment was double-blind placebo-controlled treatment with the selective noradrenaline reuptake inhibitor mirtazapine (a nontricyclic, presynaptic $\alpha_{2}$-antagonist which enhances both noradrenergic and serotonergic neurotransmission; De Boer, 1996). In case of refusal or insufficient treatment response after 8 weeks, open treatment with the selective serotonin reuptake inhibitor (SSRI) citalopram was offered (Montgomery \& Djarv, 1996). Sufficient treatment response was defined as at least $50 \%$ reduction on the Hamilton Depression Rating Scale (HDRS; Hamilton, 1960) compared with baseline score or a HDRS score at 8 weeks of $\leqslant 9$. Thus, patients who were initially treated with placebo and who did not improve within 8 weeks were subsequently treated with an SSRI. The third option was 'tailored treatment' which was at the discretion of the clinical psychiatrist (e.g. SSRI, psychotherapy, etc.). Patients were scheduled to visit the psychiatrist on average once a month during the treatment period of 6 months. In the care as usual arm, psychiatric treatment outside the study was recorded but no treatment was offered by the MIND-IT investigators. Whether the patient was referred for cardiac rehabilitation was left to the discretion of the patient's cardiologist (who was masked to the psychiatric screening results).

\section{Long-term depression status and quality of life}

At approximately 18 months postmyocardial infarction, the course and outcome of the depressive episode was assessed in a CIDI interview. The BDI was administered to evaluate the severity of depressive symptoms. In addition, health-related quality of life was assessed with the RAND 36item Health Survey, which consists of 36 items organised into eight scales (Ware et al, 1993; Essink-Bot et al, 1997). Somatic health complaints were assessed with the Health Complaints Scale (HCS), a self-report measure to assess common health complaints in patients with coronary heart disease (Denollet, 1994). Disability was assessed according to Broadhead et al (1990). Patients were asked to indicate with a time frame of the past month: 'how many days were you not able to do your daily activities (for example your work, housework, studies, leisure activities) owing to physical or emotional problems?' and 'apart from the above, on how many days were you able to do your daily activities for less than half of the time owing to physical or emotional problems?' Both complete and partial disability were categorised as having been present for either less than 1 week or for 1 week or more during the previous month.

\section{Cardiac events}

The occurrence of any significant cardiac event served as the primary end-point for the study. Cardiac events included cardiac death or hospital admission for documented non-fatal myocardial infarction, myocardial ischaemia, coronary revascularisation (coronary angioplasty or bypass surgery), heart failure or ventricular tachycardia occurring in the time between randomisation and 18 months postmyocardial infarction. Time to follow-up (6-15 months) depended on the time of randomisation (range 3 months to 12 months post-myocardial infarction). Other cardiac-related hospital admissions (defined as admissions with an initial evaluation by a cardiologist or hospitalisations at the 
cardiology ward) were considered as secondary end-points. Potential end-points were recorded at 12 months and 18 months post-myocardial infarction, and were reviewed and classified according to prespecified, established criteria (Cannon et $a l, 2001)$ by an independent end-point committee that was unaware of patients' treatment assignments. Discrepancies were discussed until agreement was reached.

\section{Statistical analysis}

Study power was calculated for long-term depression outcomes and cardiac events. With respect to the long-term depression status, a sample of 320 randomised patients would result in a study power of $80 \%$, assuming a drop-out rate of $20 \%$ and a small-to-medium effect size $(0.35)$. With respect to cardiac events, we expected a 12 month incidence of $38 \%$ for patients with depression and $19 \%$ for patients without depression (Frasure-Smith et al, 1995). If psychiatric treatment could reduce the risk for patients with depression from 38 to $25 \%$ (i.e. reduction of the attributable risk by two-thirds), 190 patients in the intervention arm and 130 in the care as usual arm would give a statistical power of 0.84 to detect this effect with a log-rank test $(\alpha=0.05)$.

$t$-tests were used to compare normally distributed continuous variables and the $\chi^{2}$-test was used to compare categorical data. Time-to-event data were analysed with the Kaplan-Meier method and differences between care as usual and intervention groups in the incidence of cardiac events were assessed with the log-rank test. Outcome data were considered at 18 months post-myocardial infarction, the time of last contact, withdrawal from the study, or at the time of a primary endpoint. All $P$ values were two-tailed.

\section{RESULTS}

A total of 4780 myocardial infarction patients were assessed for eligibility (Fig. 1). Of these, 1403 (29\%) met one or

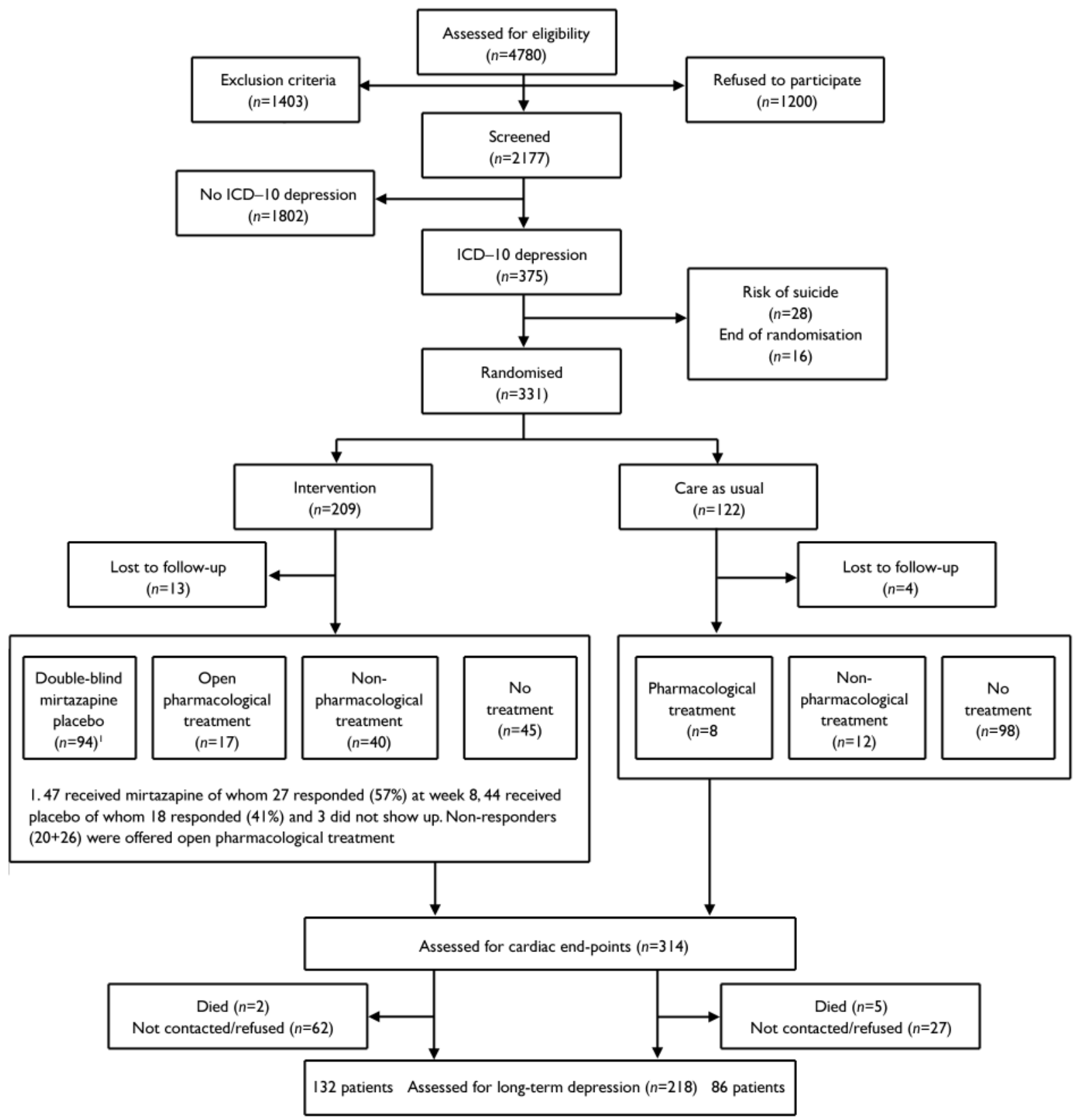

Fig. I CONSORT diagram 
more exclusion criteria, and of the excluded patients, 104 were receiving treatment for depression (see Table 1 for reasons for exclusion). Of the 3377 remaining patients, 1200 refused to participate and 2177 were included $(64 \%)$. During the screening period from 3 to 12 months post-myocardial infarction, 375 patients $(17.2 \%)$ met the ICD-10 criteria for depression. After exclusion of potentially suicidal patients $(n=28)$ and patients who were diagnosed with depression after randomisation was closed ( $n=16), 331$ patients were available for randomisation. The intervention $(n=209)$ and care as usual $(n=122)$ arms did not differ with respect to demographics, depressive symptoms during hospitalisation (BDI score), risk factors for coronary artery disease and important prognostic variables such as WMSI and comorbidity (Table 2). In addition, there were no differences with respect to ICD-10 depression characteristics (Table 3). Seventeen patients $(5 \%)$ were lost to follow-up.

\section{Antidepressant intervention}

Of the 196 patients assigned to the intervention and not lost to follow-up, 45 $(23 \%)$ did not receive antidepressant treatment, either because they refused to accept the proposed therapy or because the psychiatrist did not confirm the diagnosis of depression at the time of the visit. The median length of time from the randomisation date to the first visit to the psychiatrist was 13 days (interquartile range 7-21 days). The majority of patients in the intervention arm received clinical management of depression and $94(45 \%)$ were enrolled in the double-blind placebocontrolled medication treatment sub-study. Of these patients, 47 initially received mirtazapine and 44 initially received placebo. Three patients received no treatment because they failed to keep their appointment. Twenty patients originally treated with mirtazapine and 26 who received placebo subsequently received 16 weeks of open-label treatment with citalopram because of an insufficient response after 8 weeks of the initial treatment. The remaining patients continued to receive their original treatment. Seventeen $(8 \%)$ received immediate open-label antidepressant treatment with citalopram and $40(19 \%)$ received non-pharmacological antidepressant treatment (i.e. psychotherapy, counselling, etc.). Patients in the intervention arm who received these different treatments
Table I Reasons for exclusion of 1403 patients from trial

\begin{tabular}{lc}
\hline Reason for exclusion & $n$ \\
\hline Myocardial infarction when patient was hospitalised for another reason & 82 \\
Patient not able to communicate & 99 \\
Decreased cognitive function & 112 \\
Patient not available for follow-up/transfer to other hospital & 243 \\
Other reasons & 379 \\
Any disease likely to influence short-term survival & 87 \\
Already receiving psychiatric treatment for a current depression episode & 104 \\
Participation in other clinical trial & 297 \\
\hline
\end{tabular}

Table 2 Baseline and prognostic characteristics of randomised patients

\begin{tabular}{|c|c|c|}
\hline Variable & $\begin{array}{l}\text { Intervention group } \\
\qquad(n=209)\end{array}$ & $\begin{array}{l}\text { Care as usual group } \\
\qquad(n=122)\end{array}$ \\
\hline Age, years mean: (s.d.) & $58.6(11.5)$ & $57.5(10.6)$ \\
\hline Male gender, $\boldsymbol{n}(\%)$ & $159(76)$ & $90(74)$ \\
\hline $\mathrm{BMI} \geqslant 25, n(\%)$ & $129(62)$ & $75(63)$ \\
\hline BDI score during hospitalisation mean: (s.d.) & $11.9(7.2)$ & $11.7(6.4)$ \\
\hline Diabetes mellitus, $\boldsymbol{n}(\%)$ & $30(14)$ & $15(12)$ \\
\hline Hypertension, $n$ (\%) & $73(35)$ & $43(35)$ \\
\hline Dyslipidemia, $n$ (\%) & $174(84)$ & $103(84)$ \\
\hline Family history of CAD, $n(\%)$ & $104(5 I)$ & $62(52)$ \\
\hline Smoking, $n(\%)^{\prime}$ & $113(54)$ & $63(52)$ \\
\hline Previous myocardial infarction, $n$ (\%) & $32(15)$ & $22(18)$ \\
\hline Killip class $\geqslant 2, n(\%)$ & $28(13)$ & $18(15)$ \\
\hline Charlson (2I) category $\geqslant 3, n(\%)$ & $30(15)$ & $15(12)$ \\
\hline Q wave myocardial infarction, $n$ (\%) & $135(65)$ & $81(68)$ \\
\hline Resuscitation, $n$ (\%) & $18(9)$ & $17(14)$ \\
\hline Thrombolysis, $n$ (\%) & $72(35)$ & $55(46)$ \\
\hline PTCA, $n(\%)$ & $96(46)$ & $60(49)$ \\
\hline CABG, $n(\%)$ & $10(5)$ & $5(4)$ \\
\hline \multicolumn{3}{|l|}{ Medication at discharge, $n(\%)$} \\
\hline Acetylsalicylic acid & $172(83)$ & $109(90)$ \\
\hline Acenocoumarol & $28(14)$ & $14(12)$ \\
\hline Nitrate & $74(36)$ & $48(40)$ \\
\hline Beta-blocker & $178(86)$ & $102(84)$ \\
\hline Calcium antagonist & $39(19)$ & $24(20)$ \\
\hline Diuretics & $39(19)$ & $16(13)$ \\
\hline ACE inhibitor & $79(38)$ & $53(44)$ \\
\hline Statin & $162(78)$ & $98(81)$ \\
\hline ASAT $_{\max }$, mmol/I mean: (s.d.) & $203(175)$ & $240(235)$ \\
\hline LVEF $\geqslant 60 \%, n(\%)$ & $28(15)$ & $17(15)$ \\
\hline LVEF $45-60 \%, n(\%)$ & $79(42)$ & $49(44)$ \\
\hline LVEF $30-45 \%, n(\%)$ & $49(26)$ & $28(25)$ \\
\hline LVEF $<30 \%, n(\%)$ & $32(17)$ & $18(16)$ \\
\hline WMSI mean: (s.d.) & $\mathrm{I} .53(0.46)$ & $1.57(0.43)$ \\
\hline
\end{tabular}

BMI, body mass index $\left(\mathrm{kg} / \mathrm{m}^{2}\right)$; CAD, coronary artery disease; PTCA, percutaneous transluminal coronary angioplasty; CABG, coronary artery bypass grafting; $L V E F=$ Left ventricular ejection fraction; ASAT, serum aspartate transaminase; WMSI, Wall Motion Score Index; ACE, angiotensin converting enzyme.

I. Current smoker or stopped smoking less than 3 months. 
Table 3 Characteristics of depression in the intervention and care as usual groups

\begin{tabular}{lccc}
\hline & $\begin{array}{c}\text { Intervention group } \\
(n=209)\end{array}$ & $\begin{array}{c}\text { Care as usual group } \\
(n=122)\end{array}$ \\
\cline { 2 - 3 } & $n(\%)$ & $n(\%)$ \\
\hline $\begin{array}{l}\text { Early-onset depression' } \\
\text { Recurrent depression }\end{array}$ & $167(80)$ & $96(80)$ \\
Severity according to ICD-10 criteria & $45(22)$ & $28(23)$ \\
$\quad$ Mild & & $36(30)$ \\
Moderate & $65(31)$ & $58(48)$ \\
Severe & $98(47)$ & $28(23)$ \\
\hline
\end{tabular}

I. Within 3 months of myocardial infarction.

Table 4 Depression status and quality of life at 18 months post-myocardial infarction

\begin{tabular}{lcc}
\hline & Intervention group & Care as usual group \\
\hline ICD-I0 depressive disorder, \% & 30.5 & 32.1 \\
BDI score: mean (s.d.) & $11.0(7.5)$ & $10.2(5.1)$ \\
Complete disability ( $\geqslant 7$ days during past month), \% & 30.5 & 33.3 \\
Partial disability ( $\geqslant 7$ days during past month), \% & 28.2 & 26.7 \\
HCS score: mean (s.d.) & $13.4(9.1)$ & $14.6(9.8)$ \\
Physical health (RAND-36) score: mean (s.d.) & $39.5(6.0)$ & $39.5(5.7)$ \\
Mental health (RAND-36) score: mean (s.d.) & $44.5(8.1)$ & $43.4(8.0)$ \\
\hline
\end{tabular}

BDI, Beck Depression Inventory; HCS, Health Complaints Scale.

Table 5 Cardiac events at 18 months post-myocardial infarction

\begin{tabular}{lcc}
\hline & $\begin{array}{c}\text { Intervention group } \\
(n=196)\end{array}$ & $\begin{array}{c}\text { Care as usual group } \\
(n=I 18)\end{array}$ \\
\cline { 2 - 3 } & $n(\%)$ & $n(\%)$ \\
Cardiac death & $I(I)$ & $3(3)$ \\
Recurrent myocardial infarction & $6(3)$ & $0(0)$ \\
Revascularisation (PTCA/CABG) & $I I(6)$ & $8(7)$ \\
Heart failure & $7(4)$ & $1(1)$ \\
Myocardial ischaemia & $I(I)$ & $2(2)$ \\
Ventricular arrhythmia & $I(1)$ & $1(1)$ \\
Total & $27(14)$ & $15(13)$ \\
\hline
\end{tabular}

PTCA, percutaneous transluminal coronary angioplasty; CABG, coronary artery bypass grafting.

did not significantly differ on severity of depressive symptoms during hospitalisation (mean BDI score for those receiving doubleblind treatment 11.6 , s.d. $=6.9$; open label treatment 13.8 , s.d. $=8.7$ ); other treatment 11.6, s.d. $=6.6$; no treatment 11.0 , s.d. $=$ 6.7; $F=0.66 ; P=0.58$ ). Moreover, these patient groups did not differ significantly on ICD-10 depression characteristics.

In contrast, only 8 patients $(7 \%)$ in the care as usual arm received antidepressant

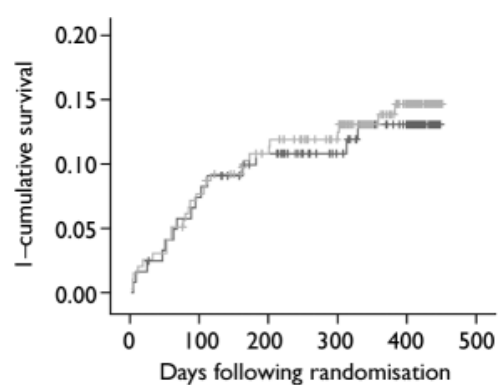

Fig. 2 Kaplan-Meier curves of cumulative probability of cardiac events for myocardial infarction patients with depression allocated to antidepressant intervention or care as usual. __ care as usual; $\ldots$, intervention; vertical ticks indicate censored data.

care as usual arm (74\%). The prevalence of ICD-10 depression was $30.5 \%$ in the patients assigned to the intervention and $32.1 \%$ in the care as usual arm $(P=0.68$; Table 4). No significant differences were observed between patients assigned to intervention or care as usual with respect to depressive symptoms, health complaints, disability and quality of life.

\section{Treatment effect on cardiac outcome}

The total event rate between randomisation and 18 months post-myocardial infarction was 42 out of 314 (13\%, Table 5). The incidence of events did not differ among the two treatment strategies $(13 \%$ in the care as usual and $14 \%$ in the intervention arm, log-rank test $0.09, P=0.76$ ). Similarly, no differences were observed in Kaplan-Meier curves (Fig. 2). The Cox regression analyses also revealed no differences between the treatment arms $(\mathrm{OR}=1.07$, 95\% CI 0.572.00). In addition, there were no differences in the incidence of cardiac events between patients in the intervention arm who received antidepressant medication (mirtazapine $n=47$; open pharmacological treatment $n=17$ ) and non-responders to placebo who received citalopram $(n=26)$ (total $n=90$ ) compared with those patients in the care as usual arm who received no antidepressant treatment $(n=98 ; \quad \mathrm{OR}=$ $0.84,95 \%$ CI $0.38-1.84 ; 14 \%$ v. $12 \%$ event rate). Within the intervention arm, the event rate for patients receiving pharmacological treatment was $13 \%$, whereas this was $15 \%$ for patients who did not receive pharmacological treatment in the intervention arm $(\mathrm{OR}=0.80,95 \%$ 
CI 0.35-1.80). There were no differences in event rates between intervention $(n=144)$ and care as usual $(n=86)$ patients with moderate-to-severe ICD-10 depression $(\mathrm{OR}=1.15,95 \%$ CI $0.56-2.38)$. Similarly, although this analysis may be underpowered, there were no differences in event rate between intervention $(n=45)$ and care as usual $(n=28)$ patients with an ICD-10 diagnosis of recurrent depression $(\mathrm{OR}=1.75$, 95\% CI 0.46-6.59).

The total rate for cardiac-related hospitalisations between randomisation and 18 months post-myocardial infarction was 127 out of $314(40 \%)$. The incidences of these secondary end-points were comparable between patients allocated to intervention and care as usual 77 of $196,39 \% v .48$ of $118,41 \%, P=0.34)$.

\section{DISCUSSION}

We found that an active treatment strategy for depression post-myocardial infarction did not improve the long-term depression status or cardiac prognosis compared with usual care. At 18 months post-myocardial infarction, about one-third of the intervention and control patients continued to have ICD-10 depression. With respect to whether cardiac prognosis can be improved by treating depression effectively, the trial is inconclusive. Our findings are consistent with the results of the ENRICHD study (Berkman et al, 2003) that showed no overall effect of CBT on the risk of all-cause mortality and reinfarction in myocardial infarction patients with depression and/or a low level of social support, and small differences in depression between the intervention and care as usual groups.

\section{Limitations}

The lack of positive results in our trial might be a result of either suboptimal antidepressant treatment in the intervention arm or better than expected treatment in the care as usual arm. This possibility is not supported by our secondary analyses because comparing treated patients in the intervention arm with untreated patients in the care as usual arm still yielded no differences in the incidence of cardiac events. However, the data needed to determine whether optimal dosage and continuation of antidepressant treatment were provided in the intervention arm were not collected.
It could also be argued that perhaps these findings are the result of a large rate of spontaneous recovery from depression which has been observed previously in both ENRICHD and SADHART clinical trials. Although this is plausible, our study was not designed to evaluate this possibility in detail since we used a Zelen design in which the care as usual patients were not informed about their depression and randomisation status. The advantage of this design is that usual care was truly representative but the disadvantage is that we cannot evaluate the (short-term) spontaneous recovery. However, the fact that both arms were comparable in depression outcomes at 18 months does support this possibility. This stresses the need to improve the identification of patients with persistent depression post-myocardial infarction in future clinical trials.

An important limitation is the power of the study. When the trial was initiated the results of the ENRICHD and SADHART trials had not been published and we had to rely on data that in retrospect may have been too optimistic (e.g. Frasure-Smith et al, 1995). First, the expected incidence of cardiac events was substantially higher than the observed incidence. Second, the association between depression and cardiac outcomes might have been overestimated and third, the anticipated effects of treatment on depression were overly optimistic. As a result, we believe that our study had sufficient power to detect differences in longterm depression outcomes (standardised effect size $>0.35$ ) but was underpowered to detect differences in cardiac outcomes. However, the nearly identical long-term depression status in the two arms and the similar rates of cardiac events offer little evidence that a significant difference would have emerged if more patients had been included. Thus, although we believe that our trial was underpowered, the observation that there were no consistent differences suggests that more study power would very likely not have yielded different conclusions.

\section{Implications}

The MIND-IT produced null findings in terms of long-term depression outcomes and was inconclusive about the effects of depression treatment on cardiac outcomes. We were unable to substantially alter long-term depression status, and as a result we still do not know whether effective treatment of depression would result in a better cardiac prognosis. Future efforts should focus on the identification and effective treatment of patients with depression post-myocardial infarction with different antidepressant treatment modalities. In the absence of such knowledge, we did not choose one specific antidepressant therapy but rather compared an active psychiatric intervention arm with care as usual. Unfortunately, this has resulted in a considerable proportion of patients that did not receive antidepressant medication although being in the intervention arm. When the efficacy of one antidepressant treatment has been proven, the next step should be to investigate whether that treatment might improve the impaired cardiac prognosis in this patient group.

Some recent findings suggest that particular subtypes of depression postmyocardial infarction might be specifically related to impaired prognosis. We have found that only somatic/affective symptoms are associated with a worsened cardiac prognosis (De Jonge et al, 2006a). Other studies have found that only incident postmyocardial infarction depression (Grace $e t$ al, 2005; De Jonge et al, 2006b) is related to poor cardiac outcomes, and that even minimal symptoms can have an effect (Bush et al, 2001). We believe that the validity and homogeneity of the diagnosis and treatment of depression in acute coronary syndromes need to be reconsidered (Frasure-Smith \& Lesperance, 2003). This might lead to treatment strategies that might be quite different from treatment for depression in the general population (American Psychiatric Association, 2000) but be better adapted to cardiac care.

\section{ACKNOWLEDGEMENTS}

We thank Robert M. Carney for comments on a previous version of this manuscript. MIND-IT is sponsored by The Netherlands Heart Foundation (97.016). MIND-IT received educational grants from Organon (The Netherlands) and Lundbeck (Denmark).

The following investigators and institutions in The Netherlands participated in the MIND-IT study: Steering committee, J. Ormel, PhD (principal investigator), A. H. Schene, MD, PhD, A. Honig, MD, PhD, H. J. G. M. Crijns, MD PhD; Study coordination, P. de Jonge, PhD, J. P. van Melle, MD PhD; Data management, Trial Coordination Centre, Groningen, The Netherlands; Clinical centres, Flevo Hospital, Almere: A. S. J. M. Sadee, MD, L. M. Konijnenberg, MD; Academic Medical Centre, Amsterdam: G. Casteelen, MD, A. M. G. Kuyper, MD, R. J.G. Peters, MD, PhD; Slotervaart Hospital, Amsterdam: M. Bax, 
MD; Nij Smellinghe Hospital, Drachten: M. van der Linde, MD, PhD; H. Teunenbroek, MD; Medical Spectrum Twente, Enschede: D. G. Buiten, MD, G. P. Molhoek, MD, PhD; University Hospital Groningen: J. A. den Boer, MD, PhD, J. F. May, MD, PhD; Tjongerschans Hospital, Heerenveen: H. P. den Daas, MD, D. G. Jochemsen, MD; Atrium Medical Centre, Heerlen: L. H. B. Baur, MD, PhD, C. J. M. van den Berg, MD, PhD; Medical Centre Leeuwarden: D. M. Tulner, MD, C. J. de Vries, MD; University Hospital Maastricht: A. Honig, MD, PhD, P. M. J. C. Kuijpers, MD, A. Schins, MD, PhD; Endpoint committee, P. M. J. C. Kuijpers, MD, PhD, J. F. May, MD, PhD, R. J. G. Peters, MD, PhD.

\section{REFERENCES}

American Psychiatric Association (2000) Practice guideline for the treatment of patients with major depressive disorder (revision). American Journal of Psychiatry, 157, I-45.

Beck, A. (1979) Cognitive Therapy of Depression. Guilford Press.

Beck, C. A., Joseph, L., Belisle, P., et al (200I) Predictors of quality of life 6 months and I year after acute myocardial infarction. American Heart Journal, 142 , 27I-279.

Berkman, L. F., Blumenthal, J., Burg, M., et al (2003) Effects of treating depression and low-perceived social support on clinical events after myocardial infarction The enhancing recovery in coronary heart disease patients (ENRICHD) randomized trial. JAMA, 289, 3106-3116.

Broadhead, W. E., Blazer, D. G., George, L. K., et al (1990) Depression, disability days, and days lost from work in a prospective epidemiologic survey. JAMA, 264, 2524-2528.

Bush, D. E., Ziegelstein, R. C., Tayback, M., et al (200I) Even minimal symptoms of depression increase mortality risk after acute myocardial infarction. American journal of Cardiology, 88, 337-34I.

Carney, R. M., Freedland, K. E., Eisen, S. A., et al (1995) Major depression and medication adherence in elderly patients with coronary-artery disease. Health Psychology, 14, 88-90.

Cannon, C. P., Battler, A., Brindis, R. G., et al (200I) American College of Cardiology key data elements and definitions for measuring the clinical management and outcomes of patients with acute coronary syndromes. Journal of the American College of Cardiology, 38, 2II42130.

De Boer, T. (1996) The pharmacologic profile of mirtazapine. Journal of Clinical Psychiatry, 57 (suppl. 4), 19-25.

De Jonge, P., Ormel, J., van den Brink, R. H., et al (2006a) Symptom dimensions of depression following myocardial infarction and their relationship with somatic health status and cardiovascular prognosis. American Journal of Psychiatry, 163, 138-144.

De Jonge, P., van den Brink, R. H. S., Spijkerman, T. A., et al (2006b) Only incident depressive episodes following myocardial infarction are associated with new cardiovascular events. Journal of the American College of Cardiology, 48, 2204-2208.

JOOST P. vAN MELLE, MD, PhD, Department of Cardiology, Thoraxcenter, University Medical Center Groningen, PETER DE JONGE, PhD, Department of Internal Medicine and Department of Psychiatry, University Medical Center Groningen, ADRIAAN HONIG, MD, PhD, Department of Psychiatry, St Lucas Andreas Hospital, Amsterdam, AART H. SCHENE, MD, PhD, ASTRID M. G. KUYPER, MD, HARRY J. G. M. CRIJNS, MD, PhD, Department of Cardiology, University Hospital Maastricht, ANNIQUE SCHINS, MD, PhD, Department of Psychiatry, University Hospital Maastricht, DORIEN TULNER, MD, Department of Psychiatry, Medical Center Leeuwarden, MAARTEN P. vAN DEN BERG, MD, PhD, Department of Cardiology, University Medical Center Groningen, JOHAN ORMEL, PhD, Department of Social Psychiatry and Psychiatric Epidemiology, University Medical Center Groningen, The Netherlands

Correspondence: Dr Peter de Jonge, Department of Internal Medicine and Department of Psychiatry, University Medical Center Groningen, P.O. Box 30.00I, 9700 RB, The Netherlands.

Email: p.de.jonge@med.umcg.nl

(First received 12 July 2006, final revision 3 January 2007, accepted 22 January 2007)

Denollet, J. (1994) Health complaints and outcome assessment in coronary heart disease. Psychosomatic Medicine, 56, 463-474.

Essink-Bot, M. L., Krabbe, P. F. M., Bonsel, G. J., et al (1997) An empirical comparison of four generic health status measures. Medical Care, 35, 522-537.

Frasure-Smith, N. \& Lesperance, F. (2003) Depression - a cardiac risk factor in search of a treatment. JAMA, 289, 3I7I-3I73.

Frasure-Smith, N., Lesperance, F. \& Talajic, M. (1993) Depression following myocardial-infarction impact on 6-month survival. JAMA, 270, 1819-1825.

Frasure-Smith, N., Lesperance, F. \& Talajic, M. (1995) The impact of negative emotions on prognosis following myocardial infarction: is it more than depression? Health Psychology, 14, 388-398.

Gillum, R. F., Fortmann, S. P., Prineas, R. J., et al (1984) International diagnostic criteria for acute myocardial infarction and acute stroke. American Heart Journal, 108, 150-158.

Glassman, A. H., O'Connor, C. M., Califf, R. M., et a (2002) Sertraline treatment of major depression in patients with acute $\mathrm{Ml}$ or unstable angina. JAMA, $\mathbf{2 8 8}$, 70I-709.

Grace, S. L., Abbey, S. E., Kapral, M. K., et al (2005) Effect of depression on five-year mortality after an acute coronary syndrome. American Journal of Cardiology, 96 1179-1185.

Hamilton, M. (1960) A rating scale for depression. Journal of Neurology, Neurosurgery and Psychiatry, 23 56-62.

Hemingway, H. \& Marmot, M. (1999) Evidence based cardiology - psychosocial factors in the aetiology and prognosis of coronary heart disease: systematic review of prospective cohort studies. BM|, 318, |460-1467.

Ladwig, K. H., Roll, G., Breithardt, G., et al (1994) Postinfarction depression and incomplete recovery 6 months after acute myocardial-infarction. Lancet, $\mathbf{3 4 3}$, 20-23.

Lane, D., Carroll, D., Ring, C., et al (200I) Predictors of attendance at cardiac rehabilitation after myocardial infarction. Journal of Psychosomatic Research, 5I, 497 501

Montgomery, S. A. \& Djarv, L. (1996) The antidepressant efficacy of citalopram. International Clinical Psychopharmacology, II (suppl. I), 29-33.
Robins, L. N., Wing, J., Wittchen, H. U., et al (1988) The Composite International Diagnostic Interview. An epidemiologic Instrument suitable for use in conjunction with different diagnostic systems and in different cultures. Archives of General Psychiatry, 45, 1069-1077.

Schiller, N. B., Shah, P. M., Crawford, M., et al (1989) Recommendations for quantitation of the left ventricle by two-dimensional echocardiography. Journal of the American Society of Echocardiography, 2, 358-367.

Schleifer, S. J., Macari-Hinson, M. M., Coyle, D. A., et al (1989) The nature and course of depression following myocardial infarction. Archives of Internal Medicine, 149, 1785-1789.

Soderman, E., Lisspers, J. \& Sundin, O. (2003) Depression as a predictor of return to work in patients with coronary artery disease. Social Science Medicine, 56, 193-202.

Strik, J. J. M. H., Honig, A., Lousberg, R., et al (2001) Sensitivity and specificity of observer and self-report questionnaires in major and minor depression following myocardial infarction. Psychosomatics, 42, 423-428.

Van den Brink, R. H., Van Melle, J. P., Honig, A., et al (2002) Treatment of depression after myocardial infarction and the effects on cardiac prognosis and quality of life: rationale and outline of the Myocardial INfarction and Depression-Intervention Trial (MINDIT). American Heart Journal, 144, 219-225.

Van Melle, J. P., De Jonge, P., Spijkerman, T. A., et al (2004) Prognostic association of depression following myocardial infarction with mortality and cardiovascular events: a meta-analysis. Psychosomatic Medicine, 66 , 814-822.

Ware, J. E., Snow, K. K., Kosinski, M., et al (1993) SF-36 Health Survey Manual and Interpretation Guide. New England Medical Center.

Watkins, L. L., Schneiderman, N., Blumenthal, J. A., et al (2003) Cognitive and somatic symptoms of depression are associated with medical comorbidity in patients after acute myocardial infarction. American Heart Journal, 146, 48-54.

World Health Organization (1990) Composite International Diagnostic Interview (CIDI).WHO.

World Health Organization (1993) The ICD-10 Classification of Mental and Behavioural Disorders: Diagnostic Criteria for Research.WHO.

Zelen, M. (1979) A new design for randomized clinical trials. New England Journal of Medicine, 300, 1242-1245. 